Pecvnia, 9 (2009), pp. 63-83

\title{
La responsabilidad del docente frente a la evaluación
}

\author{
Walter Carrizo \\ correo@unileon.es \\ Univ. Nac. de la Patagonia San Juan Bosco \\ Fac. Ciencias Económicas \\ Delegación Comodoro Rivadavia \\ Recibido: Octubre 2009 \\ Aceptado: Diciembre 2009 \\ Ciudad Universitaria $3^{\circ}$ Piso - Ruta Provincial $N^{\circ} 1 \mathrm{~s} / \mathrm{n}$ \\ 9000 Comodoro Rivadavia - Chubut (Argentina)
}

Siempre se ha tenido una idea restringida de la evaluación: ha sido asociada con el momento de la toma de exámenes. En otras palabras, equivalente a la certificación de los conocimientos que los estudiantes han aprendido y los profesores enseñado, concluyendo los procesos de enseñanza y aprendizaje.

Pero pensarla solamente como un procedimiento que constata ciertos resultados y cierra un ciclo es minimizar sus posibilidades. La evaluación puede verificar además, capacidades, competencias, habilidades y hasta actitudes, pudiendo revelar problemas tanto en el proceso de aprendizaje como en la enseñanza misma. La información resultante debería generar los mecanismos necesarios para solucionarlos y mejorar el proceso educativo en general.

Es necesario entonces, considerarla como
There has always been a misconception about evaluation: it has been associated with the moment an exam is taken. In other words, the act of evaluating is equivalent to the certification of the knowledge students have learnt and professors have taught, summarizing both teaching and learning processes.

But considering it only as a procedure which verifies certain results, as well as the simple conclusion of a cycle, minimizes the immense possibilities evaluation can offer. Capacity, performance, skill and even attitudes can also be verified in order to reveal problems in the students' learning process in addition to the teaching process. The resulting information should generate decisions which would correct those problems and enhance the education system accordingly. 
un proceso en sí misma, no exenta de complejidad, que no debe ser concebida en forma aislada de la enseñanza: se evalúa aquello que se ha enseñado, tanto en conceptos como en la metodología aplicada.

Así, la evaluación no es solamente un instrumento de certificación sino, más aún, un elemento crucial en la formación profesional de nuestros estudiantes que puede, además, retroalimentar ambos procesos de enseñanza y aprendizaje. Como formadores, nos cabe una enorme responsabilidad en la generación y comprobación de los conocimientos, que no debemos ignorar.

Palabras clave: Evaluación, Responsabilidad, Aprendizajes, Enseñanza, Resultados, Competencias, Instrumentos de evaluación.
Therefore, evaluation ought to be thought of as a process itself, quite complex indeed, which must not be considered as isolated from the teaching process: we evaluate what we teach, conceptually and methodologically speaking.

Consequently, evaluation is not only an instrument of certification but moreover, a key element in the professional education of our students, operating as feedback of the learning and teaching processes. As educators, we shoulder the responsibility of generating and verifying knowledge. Such responsibility is not to be ignored

Key words: Evaluation, Responsibility, Learning, Teaching, Results, Capacities, Evaluation Instrument.

\section{INTRODUCCIÓN}

Cuando a un docente se le pregunta en qué consiste su actividad, es seguro que se referirá al tiempo que transcurre en las aulas exponiendo teoría, ayudando a los alumnos a que resuelvan problemas y respondiendo consultas sobre aquellos puntos que generan más dificultad para su aprendizaje. Pero inevitablemente también hará mención al tiempo de espera necesario para que los alumnos resuelvan un examen parcial al momento de "evaluar" los aprendizajes, y a aquellas horas que deberá dedicar a su corrección, sin olvidar el difícil trance que suponen los exámenes finales.

En estas simples ideas podríamos diagramar un esquema lógico de nuestro desempeño: podemos rescatar un proceso de enseñanza al pensar en nuestras explicaciones, un proceso de aprendizaje de nuestros alumnos al tratar de resolver problemáticas ofrecidas, $y$, naturalmente, un proceso de evaluación de los resultados que tales aprendizajes produjeron. Huelga decir que este diagrama simplista podría suscitar interminables debates acerca de la postura que pudiéramos asumir al considerar la vinculación entre enseñanza y aprendizaje. Sin querer ser excesivamente reduccionista, se podría pensar en una concepción conductista de estos procesos, en la que el docente ofrece estímulos y el alumno responde a los mismos. Otra forma de verlos sería optar por un 
encuadre constructivista, mediante el cual el docente facilita herramientas para que el alumno procese y genere los conocimientos.

Más allá de la idea a la que se adhiera, queda aún por analizar un integrante de la secuencia lógica planteada: la evaluación, pensada como el final de un camino, como la comprobación de algo. Surgen, por consiguiente, algunas preguntas inexorables: ¿La evaluación es un punto de llegada, luego de un determinado tiempo? ¿Es así como normalmente se asume la evaluación? $i O$ es acaso un proceso más complejo, no tan natural como se pretende, en el que no solo se evalúan aprendizajes sino que también se ve involucrada la enseñanza?

En el presente escrito se centrará la atención en la temática de la evaluación como un proceso complejo que involucra más aspectos que la mera comprobación de resultados. Asimismo, se buscará contribuir a la reflexión sobre el papel que los docentes, en tanto formadores de profesionales, asumen al momento de plantearse esta crucial actividad docente.

\section{¿QUÉ PENSAMOS ACERCA DE LA EVALUACIÓN?}

Al hablar de evaluación en la educación superior, se pueden llegar a escuchar ciertas presunciones básicas tales como:

- la evaluación es un momento de comprobación de resultados en el que se cierran los procesos de enseñanza y aprendizaje. Con este pensamiento se está haciendo referencia a los momentos específicos de la toma de exámenes parciales y/o finales. Ciertamente la idea es que aquello que se ha enseñando, haya sido aprendido por nuestros alumnos. Esta acción de comprobación conlleva además la comunicación de una nota, una calificación. Si el alumno ha aprobado, pareceríamos satisfechos con nuestro actuar y se podría considerar que se ha cerrado un ciclo. Sin embargo, ¿qué ocurre si el alumno suspende? ¿Se cerró el ciclo en este caso? Podríamos pensar que aún tiene otra oportunidad con un examen de recuperación u otra fecha para rendir nuevamente el final; esto implica que el ciclo aún está abierto. También quiere decir que, como docentes deberemos preguntarnos qué se puede hacer para que en la siguiente instancia no se repitan los mismos errores y dejar al alumno librado a sus propios recursos que, posiblemente, esté aplicando mal. Es probable que nos surjan cuestionamientos tales como "con comisiones multitudinarias no se puede hacer un seguimiento de este tipo" o "mi 
cátedra no tiene los integrantes necesarios para hacer esto". Pero justamente, al darnos cuenta que algo pasa con esos conocimientos que no se reflejan con un aprobado, es que la idea de evaluación se revela como más abarcativa que el mero hecho de comprobar los resultados de los alumnos. Más aún, se puede pensar en instancias en las que se desee evaluar los conocimientos previos para constatar que ciertos aprendizajes significativos hayan sido incorporados y se pueda partir de ellos hacia una profundización posterior. Esto nos da la idea que, si el alumno aprueba, el ciclo tampoco se cierra, ya que se abren nuevas instancias de enseñanza y aprendizaje.

- la evaluación comprueba los saberes que el alumno debe tener. Esta afirmación un tanto deóntica nos posiciona como los árbitros del saber. Sin lugar a dudas que en toda disciplina existe un núcleo sine qua non de conocimientos. Nosotros, en nuestra disciplina contable, no podemos pensar, por ejemplo, en "aprobar" a un alumno si no sabe y puede aplicar el concepto de DEVENGADO. Es totalmente inadmisible. Más allá de ellos, existe un cúmulo de conocimientos que dependerán del estado del arte de la profesión, del perfil planteado por la currícula institucional y de las propias inquietudes y características del docente, entre otros. ¿Cómo estamos seguros entonces, de los conocimientos que nuestros alumnos "deben" tener? Es preciso, por lo tanto, extremar los cuidados al momento de planificar los objetivos de la enseñanza, para así poder plantear correctamente los de la evaluación. En tal caso, no solo sobre los contenidos, sino teniendo en cuenta con qué instrumento será más conveniente comprobar tales saberes. ¿Se pedirá un simple recitado del concepto para constatar que lo ha aprendido? ¿O se recurrirá al planteamiento de situaciones en las que revele capacidades específicas que le permitan aplicar tal concepto? La sentencia que abre este párrafo nos da idea de relación, de continuidad entre dos grandes procesos: la enseñanza y la evaluación, que no se pueden pensar disociadamente. Esto implica además que no se puede evaluar aquello que no se enseñó, o innovar con formatos desconocidos por el alumno, peligro que podría presentarse si quienes deciden la jerarquización de contenidos -los saberes que se deben tener- no participan en la enseñanza pero luego preparan la evaluación. Como reflexión final a este punto, quién no se ha preguntado luego, o en el transcurso de una clase "¿me entendieron los alumnos?" "¿expliqué bien?" Estos cuestionamientos no pretenden comprobar conocimientos, sino indagar acerca del desempeño propio. ¿No es esto también una instancia de evaluación? 
- evaluar implica corregir para asignar una nota. ¿Quién de nosotros no se ha enfrentado a la ardua tarea de corregir y poner notas? Nos vienen a la mente largas y aburridas horas en las que protestamos cuando encontramos esos errores que crisparían al mismísimo Lucca Paccioli. En estos casos marcaremos una cruz, lo más visible posible, para resaltar el error. Sin embargo, pensemos lo siguiente: una de las acepciones del verbo corregir es "enmendar lo errado". ¿Cómo se logra esto? No solo se debería marcar aquello que esté mal, sino, sobre todo, realizar también las aclaraciones correspondientes de manera que, si el alumno debe presentarse a una recuperación, tenga una orientación acerca de cuál ha sido su error y pueda enmendarlo. La evaluación debería ser un disparador para el aprendizaje, una instancia que permitiera encontrar la forma de subsanar equivocaciones y afianzar aprendizajes. Es difícil recordar esto cuando se tienen exámenes acumulados y un tiempo perentorio para su entrega. A pesar de ello, sería bueno no desaprovechar una oportunidad más para fijar conceptos o procedimientos. Por otro lado, existe en esta tarea de "poner notas" una implicancia aún más profunda: la idea de evaluación como acreditación de nivel. Nos enfrentamos a una dicotomía crucial en el desempeño docente: ¿evaluamos para corroborar y corregir aprendizajes o evaluamos para acreditar que un alumno ha satisfecho los objetivos del presente nivel y puede acceder al siguiente? Es evidente que en algún punto de los procesos pedagógicos sea necesario comprobar resultados y certificar conocimientos. Pero la evaluación no debería confundirse con acreditación. Si bien es un medio válido, su función no debería restringirse a cumplimentar con tal requisito académicoadministrativo. Desde otro punto de vista, asignar una nota persigue la función de transformar variables netamente cualitativas -sabe o no sabe, puede aplicar o no puede aplicar, aprueba o suspende- a una escala cuantitativa, en el afán de ser lo más objetivos posibles. Tal planteamiento nos lleva al siguiente supuesto.

- la evaluación debe ser lo más objetiva posible. Los que nos desempeñamos en la disciplina contable sabemos que la objetividad es intrínseca a la tarea de medir. Decimos por tanto, que una medición es objetiva si dos o más agentes, aplicando las mismas técnicas y procedimientos, llegan a resultados similares con un grado mínimo de desvío. El problema que se nos presenta al considerar la objetividad de la evaluación, es que mayormente, cuando tenemos que asignar notas a los exámenes, estamos solos. Dependiendo del grupo de cátedra, es probable que, cuando nos encontremos con esos exámenes de la tan temida "zona 
gris" -los dudosos- podamos recurrir a un colega para que nos ayude a definir si aprueba o no. Más aún, medir el aprobado, implica traducirlo a una escala numérica. ¿Con cuánto se aprueba su materia? Pregunta clásica de los alumnos. ¿Con 4, con 6, con 7? ¿Qué diferencias existen en el saber entre un 6 y un 7? Y una pregunta más profunda: ¿es necesario colocar una nota numérica? ¿o con un simple APROBADO o DESAPROBADO bastaría? Todas estas cuestiones ameritarían un debate más profundo y quizás una extensa jornada de trabajo para revisar las diferentes posturas al respecto. Sin embargo, algo parecería vislumbrarse. Plantear una evaluación objetiva es una tarea extremadamente difícil. Pensemos que desde el momento que somos los docentes quienes planteamos qué, cómo y cuándo evaluar, estamos involucrando nuestra propia subjetividad. Al momento de tomar un examen oral afloran también ciertas subjetividades: podemos dejarnos influir por la vestimenta, los gestos, el vocabulario. El lado positivo se nos presenta cuando, ante una duda, vemos cómo ha sido el desempeño del alumno a lo largo del curso; quizás eso defina la nota, además de los conocimientos al momento del examen. Capítulo aparte merece la confección del instrumento de evaluación. ¿Las pruebas de multliple choice son más objetivas? ¿Y las de verdadero-falso? ¿Qué ocurre cuando se ofrecen ejercicios prácticos para su resolución? Más adelante se realizará una revisión sobre las distintas alternativas de instrumentos y su aplicabilidad a la enseñanza de la contabilidad.

Todas estas consideraciones y muchas más de las que se han vertido aquí, dejan de manifiesto la complejidad de la evaluación. En ella se conjugan no solo aspectos de conocimiento sino que se entrelazan factores personales, sociales, técnicos, epistemológicos, éticos, ideológicos y político-institucionales entre otros.

Las reflexiones anteriores hicieron mayormente referencia al planteamiento de un examen, sea éste escrito $u$ oral, parcial o final. Recordemos el pensamiento de Díaz Barriga (1990) sobre esta instancia evaluadora:

(...) el examen es un espacio donde se realiza una multitud de inversiones de las realidades sociales y de las pedagógicas. (...) Es un espacio que invierte las relaciones de saber y de poder. De tal manera que presenta como si fueran relaciones de saber las que fundamentalmente son de poder (...) Los problemas de orden social: posibilidad de acceso a la educación, justicia social, estratos de empleo, estructura de la inversión para el desarrollo industrial, etc.; son trasladados a problemas de orden técnico, objetividad, validez, confiabilidad (...) 
Obviamente son cuestiones que presentan serios inconvenientes al momento de emitir un juicio de valor, como es el resultado de una evaluación. Si bien no se puede dar una solución inmediata o una receta que minimice su incidencia, es sumamente importante que se asuma su existencia y que podrían estar influyendo en nuestra práctica. La docencia es una actividad relacional. De alguna forma, queramos o no, se establece un vínculo entre profesores y alumnos. Como en toda relación, la subjetividad está presente, y por lo tanto, la confiabilidad en el vínculo. Éste debería producir acciones de ambos lados, de tal modo que nos resultara impensable la evaluación como un camino unidireccional. Los profesores evaluamos a los alumnos pero éstos también nos evalúan. Los aprendizajes y la enseñanza son objetos de la evaluación. Veamos a continuación algunas definiciones según los teóricos de educación.

\section{¿QUÉ ES LA EVALUACIÓN?}

Para P.D. Lafourcade (1977) la evaluación es:

La etapa del proceso educativo que tiene como finalidad comprobar, de manera sistemática, en qué medida se han logrado los objetivos propuestos con antelación. Entendiendo a la educación como un proceso sistemático, destinado a lograr cambios duraderos y positivos en la conducta de los sujetos, integrados a la misma, en base a objetivos definidos en forma concreta, precisa, social e individualmente aceptables.

Águeda Benito y Ana Cruz (2007) ven en la evaluación:

uno de los elementos claves del proceso formativo en cualquier nivel educativo, cuyos desarrollo y resultados tienen consecuencias en términos formativos, acreditativos e incluso económicos. (...) por lo tanto, es un proceso directamente vinculado con la calidad de la enseñanza: su correcta definición y desarrollo establecen el marco necesario para conducir el aprendizaje, para ajustar los contenidos y métodos de enseñanza y, en último término, para permitir la mejora continua del proceso formativo.

Jorge Steiman (2008) esboza una definición del término evaluación en un sentido amplio, y a partir de ella, llega al concepto de "evaluación didáctica" pensándola como:

un proceso que, a partir del conocimiento y comprensión

de cierta información, permite, desde una actitud 


\begin{abstract}
dialógica, emitir un juicio de valor acerca de las prácticas de enseñanza y/o las prácticas de aprendizaje en un contexto socioeconómico determinado en el cual se interviene con particularidad significante lo social amplio, la institución, el objeto de conocimiento, el grupo de alumnos y el/la docente y que posibilita tanto el tomar decisiones referidas a las prácticas de referencia como exige comunicar a docentes y/o alumnos/as -por medio de enunciados argumentativos- el juicio de valor emitido y las orientaciones que, derivadas de éste, resultan necesarios para la mejora de la práctica.
\end{abstract}

De estas tres definiciones se pueden rescatar pensamientos clave, tales como:

1. La visión de un proceso: tanto la evaluación como la educación en sí misma, son vistas como un conjunto de acciones que se relacionan e influyen mutuamente a lo largo de un período de tiempo. Específicamente y bajo esta visión, la evaluación se presenta como devenir continuo y no como un cierre o punto de llegada, luego del cual no hay más que hacer. La idea de coherencia e interacción nos enfrenta a una permanente retroalimentación, necesaria para el mejoramiento de todo el proceso educativo.

2. Las consecuencias que la evaluación produce: sea desde una concepción más conductistas o una más constructivista, la educación $y$, por ende la evaluación, causa efectos en las partes intervinientes y sobre los procesos de enseñanza y aprendizaje. La emisión de un juicio de valor, según Steiman, debe producir orientaciones, ajustes y correcciones que ayuden a mejorar el proceso educativo en general.

3. Si bien está presente implícitamente en todas las definiciones, Steiman resalta el carácter dialógico de la evaluación. Esto quiere decir que siempre debemos tener en mente que nuestra actividad docente se desarrolla mediante el diálogo que se establece entre profesores y alumnos. La evaluación no podría pensarse como un proceso unilateral, por el simple hecho que en la docencia se establece una relación bilateral: quien enseña y quien aprende. El mismo autor expresa "(...) Sin diálogo no es posible conocer, ni comprender, ni valorar, ni tomar decisiones".

4. En estas tres definiciones se ha mencionado comprobación de logros, ajuste de contenidos y métodos, y orientaciones derivadas de la decisión tomada. Es digno de destacar que no se ha mencionado la instancia de acreditación. Se entiende como tal al proceso de certificación 
institucional por el cual el docente, mediante la aplicación de ciertos instrumentos de evaluación, reconoce oficialmente ciertos aprendizajes por parte de los alumnos, expresados a través de una escala de valoración, en forma cualitativa o cuantitativa. Se habla aquí también de proceso, ya que la acreditación comienza con la elección de qué se solicitará a los alumnos, con qué instrumentos se les pedirá la comprobación de los aprendizajes y con qué escala se medirán los logros. De lo desarrollado podemos deducir que cada vez que debemos acreditar, estamos evaluando. Sin embargo, no siempre que se evalúa se está en presencia de una acreditación. Pensemos en las evaluaciones iniciales que se realizan. 0 en los ejercicios que se proponen en una clase a modo de comprobación de que ciertos conceptos o procedimientos hayan sido captados. La evaluación es un concepto mucho más amplio, que involucra acciones de seguimiento y comprobación, con el fin de buscar los medios de mejorar la práctica docente en general.

Evaluar por lo tanto, es asumir una actividad compleja, que pretende mejorar tanto los aprendizajes de los alumnos como los métodos de enseñanza de los docentes. De más está decir que se necesita, desde ambos lados de esta relación, tener una apertura mental que posibilite la autocrítica y, por qué no, la autoevaluación.

El planteamiento que surge es qué se evalúa (objeto), quién evalúa (sujeto), cómo se evalúa (métodos e instrumentos) y en qué momento se evalúa. Siguiendo a Steiman (2008), se puede establecer el siguiente esquema:

- Al hablar del objeto de la evaluación nos podemos referir a:

- La evaluación de la enseñanza

- La evaluación de los aprendizajes

- Si nos referimos al sujeto de la evaluación podemos pensar en:

- El docente como evaluador

- El alumno como evaluador

- El problema de los métodos e instrumentos se referirán a:

- Métodos e instrumentos de evaluación de la enseñanza

- Métodos e instrumentos de evaluación de los aprendizajes

- El momento de la evaluación dependerá si es:

- Evaluación inicial

- Evaluación de seguimiento

- Evaluación para la acreditación (parcial o final) 
Dada la amplitud de la problemática, se centrará la atención en la práctica a la que estamos más acostumbrados a pensar en términos de evaluación, es decir evaluación de aprendizajes y el docente como evaluador.

\section{¿QUÉ EVALUAMOS?}

$\mathrm{Si}$, como profesores de contabilidad, quisiéramos responder esta pregunta, podrían venir a nuestra mente opciones tales como:

- Conceptos

- Aplicación de técnicas

- Conocimiento y aplicación de la normativa vigente

- Capacidad de análisis

- Sentido crítico

- Coherencia teórico-práctica

Una pregunta que pareciera en principio tan simple de responder, encierra no tan solo connotaciones de carácter pedagógico, sino además, cuestiones más profundas, tales como posicionamientos epistemológicos que condicionarán nuestra enseñanza y, por consiguiente, el tipo de aprendizajes que se quieran evaluar.

¿Cómo asumimos la disciplina que enseñamos? ¿Es una técnica, una tecnología o una ciencia? No se abordará en este documento una discusión acerca del status epistemológico de la contabilidad. Sin embargo es preciso recordar, y sobre todo, tomar conciencia de que según sea nuestra postura al respecto, es como se encarará la enseñanza y por consiguiente, la evaluación de los aprendizajes.

Si encaramos la contabilidad como una técnica, ¿se enseñará cómo desarrollar el sentido crítico? ¿O bastará con concentrarnos en la correcta aplicación de procedimientos y herramental?

En igual sentido, existe una relación directa entre la forma en que se enseña y lo que se pretende evaluar. Si nunca se ha enfrentado al alumno a situaciones en las que debía analizar varias opciones y elegir críticamente la más conveniente, mal se le puede pedir en una evaluación que aplique tal conducta. De la misma forma, si solamente se ha enseñando conceptos sin su fundamentación y solo como un recitado, en una evaluación no se podrá pretender que el alumno justifique, por 
ejemplo, por qué en la constitución de una previsión se registra una cuenta de pérdida.

Al elegir una postura epistemológica, estamos decidiendo cómo encarar la enseñanza y, por consiguiente qué evaluar. Queda aquí de manifiesto la estrecha relación que se establece entre estos dos grandes aspectos de nuestra práctica docente y de la que debemos ser conscientes, dada la responsabilidad que nos cabe como formadores de futuros profesionales.

Pero la pregunta acerca de qué evaluamos, puede referirse también a si evaluamos resultados como producto o nos centramos en el desempeño de los alumnos. Este nuevo planteo condiciona todo el proceso de evaluación y su estrecha relación con el de enseñanza. Al hablar de evaluación de resultados, nos viene a la mente la idea de etapa final, de cierre de ciclo. Es quizás aquí donde evaluación y acreditación coinciden: los instrumentos que se utilizan suelen pensarse como herramientas "de constatación y de medición del aprovechamiento del alumno, tras un período más o menos prolongado de enseñanza" (Benito y Cruz 2007: 89), que se traduce en una calificación numérica que certifique los progresos de los estudiantes. Posiblemente esto nos resulte familiar al plantear los exámenes parciales y finales.

Cuando se hace referencia a la evaluación de desempeño, surge la idea de un continuo en el tiempo, de la observación de la actuación del alumno en situaciones ficticias o reales. El desempeño se evaluará según el "saber" del alumno, su "saber hacer" y el "saber ser" en tales situaciones. Es decir que se pretenderá evaluar ciertas competencias del estudiante en relación a una disciplina o un conjunto de ellas, de las que no solo tendrá que aplicar conceptos, sino además procedimientos y actitudes. Se ponen en juego así, cualidades personales que se interrelacionarán con el entorno en el que se debe actuar para lograr ciertos resultados. Como se dijo anteriormente, el alumno debe "hacer" en un entrono real o próximo a tal condición. Es probable que este concepto nos resulte poco aplicable, o restringido a asignaturas del ciclo profesional en las que el estudiante está más en contacto con la realidad profesional. Sin embargo, no debemos olvidarnos que la universidad forma profesionales, y el camino de la formación comienza desde el primer día que los ingresantes de primer año asisten a una clase. Es un camino que deben transitar con la guía de los docentes, en nuestra área todos contadores, que saben hacer y ser profesionales. Por lo tanto, no es de descartar la idea desde los primeros años de carrera, máxime teniendo en 
cuenta que mundialmente la educación ha virado hacia la formación a través de capacidades y competencias, es decir, el conocimiento aplicado a la solución de problemas de la vida real. Nuestros alumnos, al egresar, ¿están capacitados para desempeñarse como profesionales? Es una pregunta que atraviesa transversalmente todo el plan de estudio de una carrera.

De esta forma, estaríamos hablando de una evaluación para el aprendizaje y no solamente la evaluación de los aprendizajes, como sugiere Philippe Perrenoud (2008). Si bien el cambio de preposiciones ("para" por "de") parece sutil, encierra un profundo cambio de visión. La información recabada en la evaluación debería ser comunicada al alumno, de modo que se sienta partícipe de lo actuado. Los errores deberían ser analizados de manera que se puedan desentrañar problemas en el aprendizaje y/o en la enseñanza, produciendo las correcciones necesarias a ambos procesos. La evaluación debería servir para aprender cómo mejorar la educación en general. Vuelven aquí a aparecer cuestiones que ya se habían mencionado al inicio de este escrito, tales como: "con comisiones multitudinarias no se puede hacer un seguimiento de este tipo" o "mi cátedra no tiene los integrantes necesarios para hacer esto". Pero, ¿nuestra función de formadores no merece el esfuerzo de lograr algún cambio? Siempre una aproximación, por pequeña que sea, es mejor que nada.

\section{¿CÓMO EVALUAMOS?}

Haremos referencia a continuación a los métodos y los instrumentos que se aplican al momento de evaluar. Para comenzar a dar respuesta a esta pregunta, se debe recordar en primer lugar, que la evaluación dependerá de la metodología de enseñanza que se haya aplicado. Deberá tenerse siempre presente la coherencia que debe existir entre lo enseñado y lo que se pide en una evaluación. A modo de ejemplo, si se ha enseñado la partida doble sólo como una técnica cuyo funcionamiento y aplicación se ha memorizado como pasos a seguir, no se podrá pedir fundamentación y relación con la ecuación patrimonial y su correspondencia con los conceptos de financiación y aplicación.

Además, como se ha expresado en el apartado anterior, según se quiera evaluar resultados o desempeños, los instrumentos que se diseñen tendrán características diferentes. Recordemos que también se pueden medir resultados, no con una visión de punto de llegada, sino como fortalecedores de los aprendizajes. 
Comencemos por los instrumentos para evaluar resultados. Existe una amplia variedad de ellos que, probablemente, todos hayamos aplicado a lo largo de nuestro desempeño. Podemos citar entre ellos:

- Exámenes orales:

- Individuales

- Grupales o de coloquio

- Exámenes escritos:

- De desarrollo estructurado:

* De elección múltiple o "multiple choise"

* Verdadero-falso

* Para completar

* De respuesta corta

- De desarrollo semiestructurado

- De desarrollo abierto

Las evaluaciones orales son constantemente cuestionadas por los especialistas en ciencias de la educación. Se los considera poco válidos y poco objetivos, ya que pueden intervenir variables que no se relacionan directamente con los aprendizajes a evaluar. El docente puede verse influido por los rendimientos anteriores que haya alcanzado el estudiante, su aspecto físico -forma de vestir y de desenvolverse en el examen- y su desempeño lingüístico. Alicia Camilloni (1989: 146) hace referencia a un "efecto de halo" que se crea alrededor de cada alumno, y que lleva al docente a formarse "una impresión o juicio sobre una característica particular de un individuo, basada sobre la impresión general que se tiene de él. Si la impresión general es favorable, será hipercalificado en los rasgos positivos y viceversa". Sin embargo Steiman (2008: 173) sostiene que el examen oral "resulta un buen instrumento si la interrogación ayuda a que el alumno/a pueda ir elaborando un discurso en el que las relaciones conceptuales se vayan encadenando naturalmente a partir del desarrollo de las ideas eje...".

El modo oral se asocia especialmente a los exámenes finales, en los que la carga emocional es muy grande y la gran mayoría de los alumnos sienten que van a un juicio sin defensor. Un intento por dar solución a este tipo de exámenes es aplicar el sistema de promoción directa sin examen final, es decir a través de exámenes parciales escritos en los que se evalúa teoría y práctica, y cuya aprobación total acredita los aprendizajes. Esta metodología debe ser muy bien planificada, ya que podría caerse en la tentación de utilizarla para eludir las largas jornadas 
de finales. Varios teóricos, incluido Steiman, no se expresan favorables a la promoción directa. Sostiene que el examen final es la instancia en la que el alumno hace una integración general de los conceptos y sus interrelaciones, de manera tal que es el momento en el que logra ver el conjunto de aprendizajes. A esta postura podríamos agregar el hecho que muchas profesiones, incluida la nuestra, necesitan de la oralidad, ya que el profesional debe defender su postura ante escenarios diversos. ¿No es acaso el examen oral una instancia dentro de tal formación?

La alternativa al examen individual es el grupal o de coloquio. En este caso es necesario tener un muy buen manejo de grupo, de modo que todos y cada uno de los integrantes sean evaluados. Esta modalidad se puede aplicar en aquellas asignaturas que requieran de la aplicación teórico-conceptual a situaciones planteadas a través de casos o metodología de taller. Sirven además, para comprobar aspectos actitudinales, tales como responsabilidad, adaptación al trabajo en grupo, manejo de los tiempos, entre otros. Se podría pensar en asignaturas como Auditoría o Practica Profesional. Sin embargo, los trabajos de campo mediante casos pueden plantearse ya desde primer año, por ejemplo, para el diseño del sistema contable de una empresa.

Los exámenes escritos también son susceptibles de críticas acerca de su confiabilidad y su objetividad. En aras de fortalecerlos en tales aspectos, se han ideado alternativas cuyo fin es reducir la subjetividad del docente al momento de su corrección. Es el caso de las pruebas estructuradas, entre las que sobresalen aquellas que se plantean de opción múltiple o "multiple choice". Este tipo de instrumento se utiliza para evaluar conocimiento, comprensión y juicio. Sin embargo deben extremarse los cuidados al momento de su confección. Si se desea utilizarlo como recurso único, se aconseja que una prueba de opción múltiple cuente entre no menos de 80 preguntas ni más de 100. Los expertos consideran que con tales cantidades se pueden evaluar todos los puntos de una asignatura o área de conocimiento. Más allá del número, es necesario tener en cuenta que, al ser de opción múltiple, cada pregunta debe contar con varias alternativas, lo aceptable son 4 , una de ellas la correcta y las restantes son distractores en los que no se debe inducir la respuesta o confundir al alumno con datos inútiles o mal escritos, ya que caeríamos en subjetividad de armado de evaluación.

Las pruebas de verdadero-falso pretenden evaluar lo esencial y útil ya que consideran que todo conocimiento se puede reducir a una proposición. Su inconveniente es que en casi todas las áreas del 
conocimiento, son pocos los aspectos que no tengan matices u opciones. Se considera además, que favorecen el aprendizaje memorístico y que están sujetos a cierto margen de azar, ya que los alumnos pueden recurrir a adivinar la respuesta.

Las pruebas con preguntas para completar buscan que el alumno concluya una frase en la que se han dejado claros en los que se centra el problema a resolver. Buscan, principalmente, evaluar la memoria.

Las pruebas de respuesta corta evalúan conocimiento y memoria, pudiendo ser usadas para corroborar coherencia y relación, al preguntar "¿por qué?" o pedir justificar una situación simple.

¿Cuál es la aplicabilidad de todos estos tipos de instrumentos a la evaluación del conocimiento contable? Como en nuestra disciplina es sumamente importante la puesta en práctica de los conceptos y la formación de criterio, estas modalidades presentan una gran limitación. Podrían plantearse, en forma única o una combinación de ellos, para evaluar aspectos teóricos. Pueden además utilizarse para realizar evaluaciones diagnóstico o a lo largo del curso. En estos casos suelen ser útiles ya que revelan "puntos flojos" en la teoría. Debe recordarse que su confiabilidad está afectada por el margen de adivinación que apliquen los alumnos. Además, si bien ahorran mucho tiempo al momento de corregir, como se puede ver a través de las características de cada uno de ellos, son los armadores de la prueba quienes llevan la mayor carga. Son ellos quienes deben confeccionarlos de manera tal que las consignas no sean ambiguas o induzcan premeditadamente a error. Como se ve, no es tarea fácil.

Las pruebas semiestructuradas constan de una presentación de un problema y de preguntas a resolver sobre el mismo. Esta estructura es la clásica aplicada a las evaluaciones prácticas en contabilidad. Se plantea una situación y ciertos interrogantes que el alumno debe responder mediante registraciones $\mathrm{y} / \mathrm{o}$ aplicación de criterio. Para ello, debe constar de una base conceptual que le permita discernir qué registración hacer o a qué conceptos recurrir para tomar un determinado criterio. Nuevamente aquí quien prepara el examen debe tener en cuenta que los enunciados no adolezcan de:

- Ambigüedad de las consignas

- Imprecisión en la redacción

- Datos incompletos

- Libertad de los alumnos para suponer situaciones 
Las preguntas deben tener una respuesta precisa. De existir varias respuestas posibles, el margen de dispersión debería acotarse con la correcta fundamentación de los conceptos involucrados.

Las pruebas de respuesta abierta se aplican a aquellos casos en los que se requiere que el alumno escriba sobre un tema específico y que él mismo le dé la estructura correspondiente. Tiene la desventaja que evalúan un único punto, no son de aplicación general y la corrección suele ser subjetiva. Como ejemplo valgan las monografías que se solicitan, sobre todo en las asignaturas de los últimos años de carrera, tal el caso de las que se relacionan con temáticas tributarias.

Más allá del instrumento de evaluación que se elija, es importante resaltar la responsabilidad que le cabe al equipo de cátedra que lo aplicará. Debe tenerse en claro, como primer paso, cuáles son los objetivos de la evaluación, es decir ¿QUÉ SE EVALÚA? ¿memoria? ¿razonamiento? ¿relación? ¿criterio? Asimismo, la redacción es sumamente importante ya que, de ser imprecisa o ambigua, el alumno podría pensar que existe "una trampa" y pretenderá adivinar las intenciones del docente, perdiendo tiempo en la resolución o adivinando la respuesta. En cátedras numerosas, en las que varias personas corrigen, se presenta el problema de la unidad de criterio en la corrección y asignación de puntuación. Es necesario establecer en tal caso, una clave de corrección previa así como la escala de puntuación, de modo que se reduzca la subjetividad. Pero sobre todo, la evaluación debería servir al alumno para que éste corrobore sus aprendizajes y aprenda de sus errores. Es imperiosa la devolución y la explicación de las equivocaciones. Esto permitirá también detectar algún problema en la enseñanza.

La evaluación de desempeño requiere de una tarea de observación de ciertas destrezas que el alumno ha debido adquirir a lo largo del curso. Lógicamente, este tipo de evaluación deberá estar precedida por una metodología de enseñanza que haga demostraciones didácticas de las destrezas requeridas, dentro de las competencias de acción que debe tener un futuro egresado.

La observación puede o no requerir de una nota. Es decir que estamos ante una metodología que no necesariamente esté acompañada de la acreditación de la asignatura, sino que servirá, sobretodo, para constatar cuál es el grado de avance en los conocimientos, y si las capacidades necesarias han sido adquiridas. Es muy útil además, para detectar dificultades tanto en el proceso de aprendizaje como en la 
enseñanza misma, pudiendo trabajar inmediatamente sobre los errores detectados.

Existen varias técnicas que trabajan este análisis concomitante. Entre ellas se pueden citar:

- El portafolio: los alumnos van acumulando trabajos que ya han sido tratados y corregidos. Al momento de la evaluación se solicita que elija un número reducido de entre ellos que considere los mejores y que comente por qué los eligió, qué aprendizajes rescata y qué aportes puede hacer sobre el tema. Parece difícil pensar en su aplicación a la evaluación de la disciplina contable. Sin embargo, una asignatura que verse sobre Análisis de Estados Financieros podría plantearse con la metodología de taller. En ella se podría requerir que los alumnos vayan acumulando estados financieros sobre los que practiquen las técnicas de análisis a medida que avance el curso. Al final del mismo se podría pedir que expusieran y defendieran aquel que consideren su mejor trabajo.

- La técnica de ECEO: la sigla corresponde a ESTRUCTURAS, COMPETENCIA, EXAMEN, OBJETIVOS. Se crean situaciones simuladas de la realidad, que se disponen en forma de circuito y por el que todos y cada uno de los alumnos deben pasar. Se solicita la resolución de una situación en un plazo no mayor a 10 minutos, tiempo en el que deberá demostrar no sólo conocimientos sino además, ciertas destrezas específicas para el desempeño de la profesión. Es ideal para las asignaturas relacionadas con la Práctica Profesional, por ejemplo, situaciones para resolver cuestiones impositivas, consultas de gerencia, etc.

- La técnica MINI CEX (CLINICAL EXAMINATION): en ésta, se enfrenta al alumno a una situación real, en un entorno de práctica auténtica. La observación no durará más de 20 minutos, pero deberá repetirse varias veces a lo largo del curso. Es un recurso apto para evaluar las prácticas o pasantías que los alumnos realicen en empresas reales.

En estas técnicas es de extrema importancia plantear los objetivos y los criterios de evaluación. Miden básicamente la experticia que va desarrollando el alumno a lo largo de un proceso y, por consiguiente, necesitan de evaluadores expertos en las temáticas abordadas. 


\section{¿CUÁNDO EVALUAMOS?}

El momento de la evaluación depende en cierta forma, de aquello que se quiera evaluar. Es decir: ¿Necesitamos saber si los aprendizajes significativos que requerimos como base de nuestra asignatura están afianzados? ¿Necesitamos saber cómo progresan nuestros alumnos? ¿Necesitamos saber si los conocimientos que posee el alumno lo habilitan para que acredite la materia? Esta "necesidad de saber", de tener información acerca de los aprendizajes de nuestros alumnos, es la que nos lleva a plantear diversos momentos y diversos instrumentos. Es así que se pueden plantear evaluaciones:

- De diagnóstico: se realiza al inicio del curso y brinda información sobre los aprendizajes de los alumnos, necesarios para una nueva etapa. En esta instancia se pueden utilizar diversos instrumentos. Por ejemplo, para cursar Auditoría, es necesario tener una buena base conceptual y de procedimientos ya afianzados, para poder acceder al análisis de la herramienta propio de la materia. En este caso puede ser útil una prueba de opción múltiple, 0 una serie de ejercicios semiestructurados en los que el alumno debe demostrar la aplicación de tales temas.

- De seguimiento: es la denominada evaluación concomitante. Ésta puede ser estructurada o no. Puede estar acompañada de calificación o no. Generalmente no se la asocia con la acreditación, aporta información acerca de los avances de los alumnos en cuanto a conocimientos y destrezas y los instrumentos a aplicar de penderán de si es:

* Estructurada: puede ser una instancia de acreditación o no. Si se están evaluado capacidades, se podrán aplicar técnicas tales como ECEO o MINI CEX, o el sistema del portafolio. Si se evalúan resultados, las pruebas escritas de nuestros exámenes parciales son un buen ejemplo. En este último caso, para que sirva como correctora de errores, sus resultados deben ser comunicados a los alumnos y revisados y discutidos los errores y las problemáticas por las que se cometieron.

* No estructuradas: surgen de la observación cotidiana que el docente realiza sobre su propia clase al preguntarse ¿entendieron lo que expliqué? Probablemente se plantee un ejercicio práctico sencillo e inmediato para tal constatación. Es el docente quien debe tener una amplitud de visión y criterio que le permita detectar problemas no solo en los aprendizajes, sino además en la enseñanza. 
- De acreditación parcial o final: están referidas a los exámenes parciales y finales que pretenden cerrar etapas en el aprendizaje. Como ya se dijo, este tipo de evaluación, si bien necesaria, será mucho más útil si el alumno puede aprovecharla como otra instancia para aprender y no como un obstáculo a sortear para obtener el título.

\section{ALGUNAS APRECIACIONES FINALES}

Como se puede ver, existe una amplísima gama de instrumentos de evaluación. Asimismo, el momento de evaluar no se restringe a la toma de parciales o al final del curso. Ambos aspectos además, están íntimamente relacionados con el proceso de enseñanza, al que avocamos tantos esfuerzos: dependiendo de cómo enseñamos, deberá ser la evaluación.

Se entiende así a la evaluación, como un proceso complejo, que no se resume a la redacción de un examen escrito y a asignar notas. Desde ya que estos pasos están presentes, pero se corresponden a una instancia de acreditación, que forma parte del proceso en general. Es así sumamente importante tener en claro qué se va a evaluar: ¿Resultados? ¿Destrezas? ¿Memoria? ¿Sentido crítico? Las destrezas, la memoria y el sentido crítico, ¿se enseñaron durante el curso?

Como ya se ha expresado repetidas veces a lo largo de este escrito, la evaluación debería favorecer el aprendizaje y no solamente medirlo. Esto implica mayor tarea para el docente, quien deberá realizar un seguimiento de sus alumnos para entender cómo aprenden y cuáles son los problemas que se les presentan en tal proceso. La información recabada luego de la prueba, debe ser devuelta con el propósito de analizar los errores, detectar dificultades y sugerir cursos de acción. Puede ser que en varios casos el consejo vertido al alumno sea "Hay que estudiar más", pero aún así es bueno entablar el vínculo con el estudiante. La idea básica es que la evaluación no debería resumirse a la comunicación de ciertos resultados sin aprovecharla para sugerir las correcciones que se pueden hacer.

Susana Camilloni et al. (1998: 43) resumen estas ideas al decir que:

Si el docente logra centrar su atención en tratar de comprender qué y cómo están aprendiendo sus alumnos, en lugar de concentrarse en lo que él les enseña, se abre la posibilidad de que la evaluación deje de ser un modo de constatar el grado en que los estudiantes han captado 
la enseñanza, para pasar a ser una herramienta que permita comprender y aportar a un proceso.

Por otro lado una cuestión que preocupa a todos los docentes $y$, por supuesto, a los alumnos, es la objetividad de la evaluación, aunque es de suponer que tal objetividad está mayormente centrada en la calificación que se asigne al examen. Como se ha visto, es prácticamente imposible asegurar una objetividad absoluta. Lo subjetivo está presente en toda instancia: desde la fijación de los objetivos a la decisión de la escala de medición a utilizar, desde la elección y la confección del instrumento de evaluación a las apreciaciones personales que todo docente tiene acerca de su grupo de estudiantes.

Lo recientemente expresado nos lleva a pensar en la gran responsabilidad que nos cabe como docentes formadores al momento de plantear la evaluación. Reflexionemos sobre lo siguiente: Los profesionales que reciban su título deberán ser capaces de desempeñarse en sus actividades con los conocimientos, habilidades y destrezas que nosotros hayamos enseñado. Por tal motivo ¿cómo no sentirnos responsables al preparar un examen? Pensando qué queremos evaluar, armando los instrumentos con consignas claras, sin ambigüedades, sin claros para que el alumno suponga algo, en fin, que la evaluación sea confiable en cuanto a su propósito, cual es mejorar la educación en general.

Más aún, el alumno también aprende con nuestro ejemplo. Como actuemos ante ellos es la idea de profesional que se formarán. La enseñanza, por lo tanto, traspasa los límites de los libros. Nuestra sola presencia deja una impronta en ellos. Este es un tema que nos lleva a pensar que nosotros como docentes profesionales estamos constantemente siendo evaluados por nuestros alumnos: desde el momento que entramos al aula, comienzan las apreciaciones hacia nuestra persona, nuestra metodología y, por supuesto, hacia la forma de evaluar que tengamos. Nosotros y nuestra forma de enseñar nos convertimos en objeto de evaluación, acrecentando aún más nuestra responsabilidad.

La Facultad de Ciencias Económicas de la Universidad Nacional de Salta -Argentina- ha sido la encargada, en el año 2009, de organizar las XXX JORNADAS UNIVERSITARIAS DE CONTABILIDAD en dicho país, bajo el lema "LA SABIDURÍA VIENE DE LA UNIVERSIDAD". Se entiende como sabiduría aquel conocimiento profundo que se obtiene a través del estudio y la experiencia. Es precisamente nuestra responsabilidad generar tal conocimiento en nuestros alumnos, el que, luego de años de estudio, se 
convertirá en el activo intangible más importante al momento del desempeño profesional. Es así que la evaluación, como elemento integrante del proceso formativo, cumple una función crucial al permitir no solo certificar los conocimientos sino, sobre todo, retroalimentar tanto los aprendizajes como la enseñanza misma, profundizando así los conocimientos, logrando así que la universidad cumpla con su cometido: generar sabiduría. Nosotros, como integrantes de la universidad, debemos velar por que tal misión se cumpla.

\section{BIBLIOGRAFÍA}

AusubeL, D.P.; J.D. NovaK y H. HANESIAN (2000) Psicología educativa: Un punto de vista cognitivo, $2^{a}$ ed., México: Trillas.

BENITO, Águeda y Ana CRUZ (2007) Nuevas Claves para la Docencia Universitaria. Madrid: Editorial Marcea.

CAMILlONI, Alicia (1989) Las apreciaciones personales del profesor. Buenos Aires: CEFyL UBA.

-, Susana CelmA, Edith LITWIN y M. del Carmen PALOU DE MATÉ (1998) La evaluación de los aprendizajes en el debate didáctico contemporáneo. Buenos Aires: Editorial Paidós.

Carretero, Mario (2009) Constructivismo y Educación. Buenos Aires: Editorial Paidós.

DíAZ BARRIGA, Ángel (1990) Currículum y Evaluación Escolar. Buenos Aires: Cuadernos Rei Argentina. IEAS-Aique.

FINKELSTEIN, Claudia (2009) Seminario: Evaluación de los Aprendizajes de los alumnos en la Universidad. Material didáctico no publicado. Comodoro Rivadavia (Argentina): Universidad Nacional de la Patagonia San Juan Bosco.

LAFOURCADE, P.D. (1977) Evaluación de los Aprendizajes. Madrid: Editorial Cincel.

PeRRenOUd, Philippe (2008) La evaluación de los alumnos. De la producción de la excelencia a la regulación de los aprendizajes. Entre dos lógicas. Buenos Aires: Editorial Colihue.

StEIMAN, Jorge (2008) Más Didáctica (en la Educación Superior). Buenos Aires: UNSAM edita. 